Case report

\title{
Choroidal neovascular membrane: Report of pediatric idiopathic case.
}

Saadouli Dorsaf ${ }^{1,3,{ }^{* *}}$, Ben Mansour Khaoula 1,3, Zouara Haifa, Yahyaoui Salem ${ }^{2,3,}{ }^{*}$, Mahdhi Sameh ${ }^{1,3}$, El Afrit Mohamed Ali 1,3.

1: Department of ophthalmology La Rabta Hospital, Tunis, Tunisia

2: Department of Pediatrics C, Children

Hospital, Tunis, Tunisia

3: College of medicine Tunis Tunisia

* Corresponding author

** Academic Editor

Correspondence to:

yahyaouisalem@yahoo.fr

Publication Data:

Submitted: August 28,2020

Accepted: October 30,2020

Online: November 30,2020

This article was subject to full peerreview.

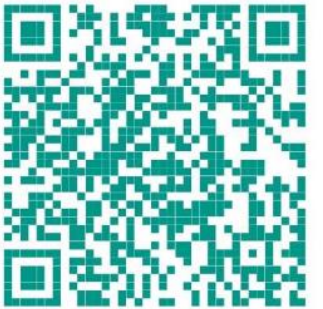

This is an open access article distributed under the terms of the Creative Commons Attribution NonCommercial License 4.0 (CCBY-NC) allowing sharing and adapting.

Share: copy and redistribute the material in any medium or format.

Adapt: remix, transform, and build upon the licensed material.

the work provided must be properly cited and cannot be used for commercial purpose.

\section{Abstract}

The choroid neovascular membrane is a rare cause of central vision loss among children. Its etiopathogenesis is still unclear and the management is not standardized. The neovascularization may be due to inflammatory, post-traumatic, or dystrophic causes. We described herein a case of choroidal neovascular membrane in a 14-year-old boy with no evident origin after investigations.

Keywords:

choroidal neovascular membrane, idiopathic, child.

\section{Introduction}

Choroidal neovascularization (CNV) in children is a rare entity which may lead to serious visual impairment [1]. In literature, its association with inflammatory, infectious, dystrophic, and traumatic disorders has been always reported. However, their etiopathogenesis is still unclear [2]. Several therapeutic options are available, but the treatment is still case-based and usually extrapolated from the adult patient's management.

In this work, we propose to highlight the clinical, therapeutic and prognostic characteristics of $\mathrm{CNV}$ in pediatric practice.

Observation

A 14-year-old female without previous medical history presented with progressive bilateral blurry vision. On examination, she had a sciascopy of +1.5 diopter at the right eye and +1 diopter at the left one. The best corrected visual acuity in both eyes was $4 / 10$ on the Snellen scale.

Anterior segment examination by slit lamp was normal. Intraocular pressures were normal. The examination of the fundus showed a greyish macular bilateral lesion suggestive of a neovascular membrane (Fig1). Fluorescein angiography showed an early macular dye leakage increasing at the intermediate phase and persisting at the late phase (fig 2). Macular optical coherence tomography scan (OCT) showed a bilateral hyperreflective fibrovascular complex located above retinal pigment epithelium layer (fig 3). OCT-A scan revealed a glomerular pattern CNV at the right eye and a tree-like pattern at the left eye (fig 4). Exhaustive etiological assessment was performed. The complete blood count, the erythrocyte sedimentation rate, the CRP, the renal and liver function were normal. Quantiferon and tuberculosis test were negative. We eliminated traumatic and iatrogenic causes.

Serological tests of syphilis, toxoplasmosis, cat scratch disease, herpes and cytomegalovirus were negative. Hemostasis analysis and hemoglobin electrophoresis were also normal. Best's disease complicated by new vessels was unlikely as the family history and the results of electrophysiological exploration (fig $5 \mathrm{a}$ and $\mathrm{b}$ ) were negative. The general pediatric examination did not reveal any abnormalities. Finally, we retained the diagnosis of bilateral idiopathic retro foveolar choroidal neovascular membrane. Intravitreal injection of anti VEGF and photodynamic therapy (PDT) were considered. 


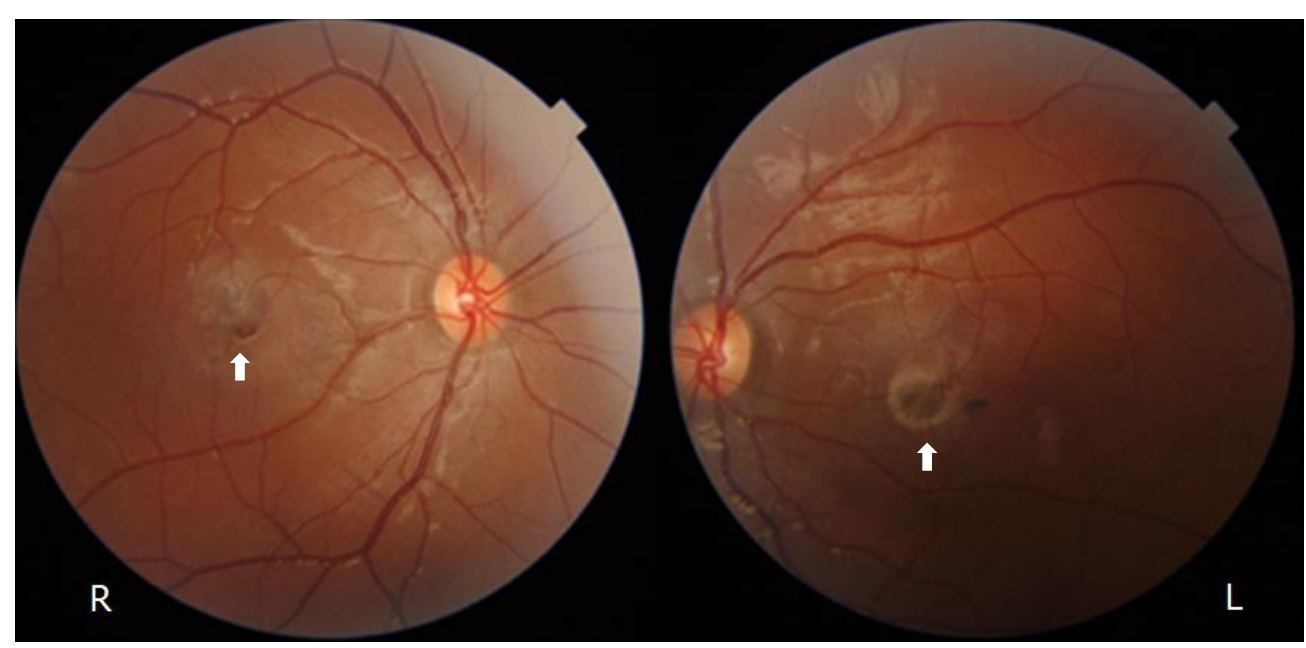

Figure 1: Fundus photography suspected macular bilateral lesion making $1 / 2$ papillary diameter suggestive of a neovascular Membrane.
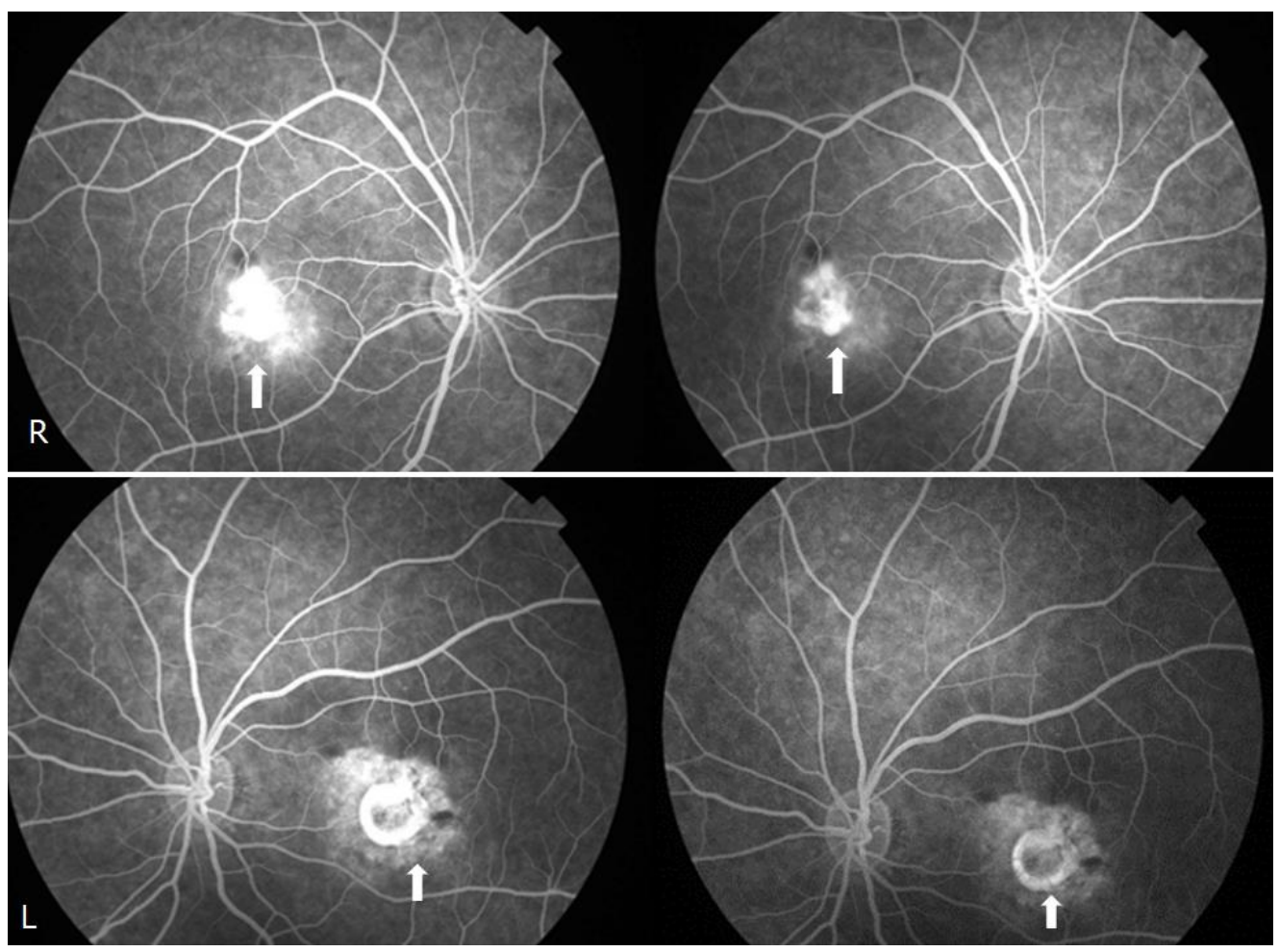

Figure 2: Fluorescein angiography (FA) showed an early persistent macular dye leakage

Citation: Saadouli D, Ben Mansour K, Zouara H, Yahyaoui S, Mahdhi S, El Afrit MA. Choroidal neovascular membrane: Report of pediatric idiopathic case. Jr.med.res.2020;3(3): 15-19. Abid et al @ All rights are reserved.

Submit your manuscript: $\underline{w w w}$.jmedicalresearch.com 


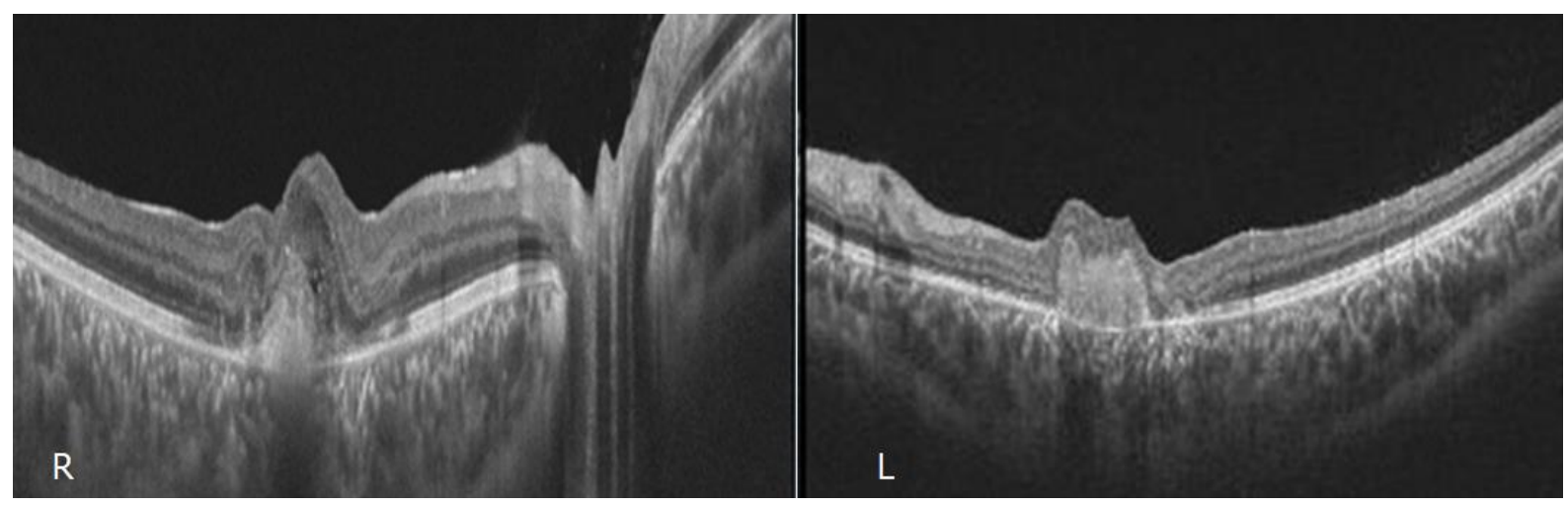

Figure 3: Linear horizontal trans-foveolar scan (Spectral HD OCT) showed a bilateral hyperreflective fibrovascular complex located above retinal pigment epithelium layer.
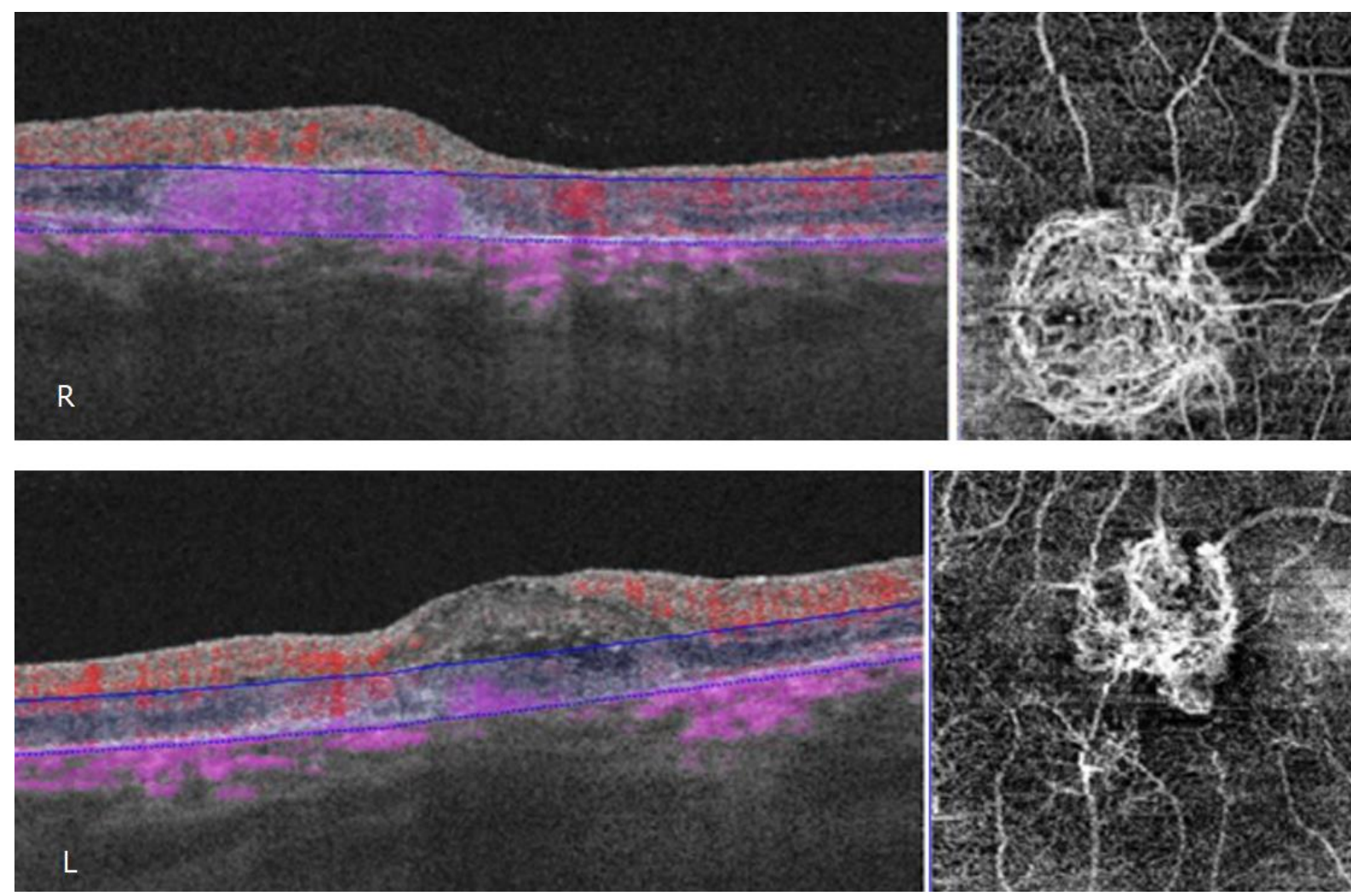

Figure 3: OCT-A scan revealed a glomerular pattern CNV at the right eye and a tree-like pattern at the left eye.

Citation: Saadouli D, Ben Mansour K, Zouara H, Yahyaoui S, Mahdhi S, El Afrit MA. Choroidal neovascular membrane: Report of pediatric idiopathic case. Jr.med.res.2020;3(3): 15-19. Abid et al @ All rights are reserved. 

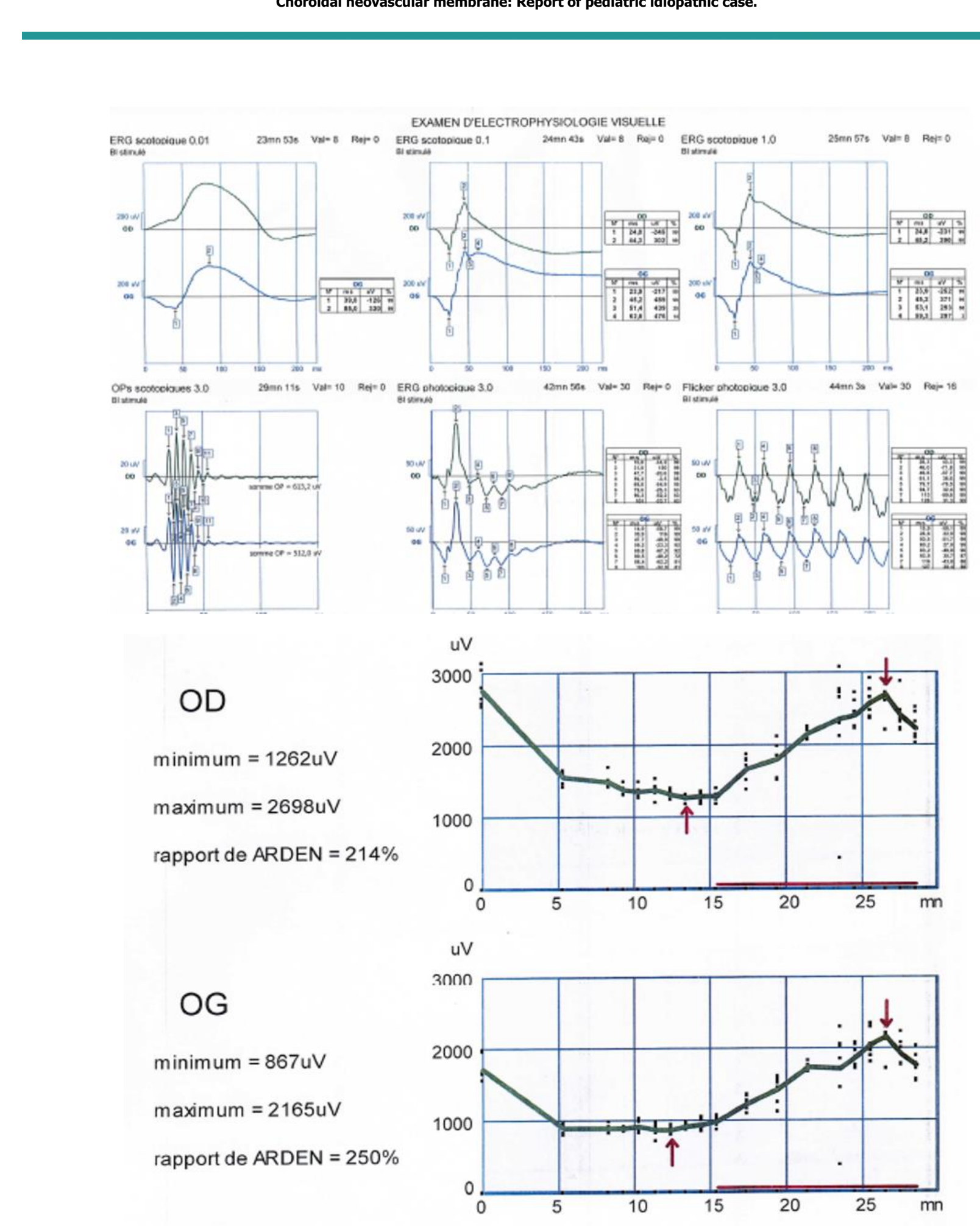

Figure 5: Electrooculogram 


\section{Discussion}

Choroidal neovascularization (CNV) is a neoformation of capillaries penetrating the Bruch's membrane, the retinal pigment epithelium and later potentially into the subretinal space [1]. Pediatric choroidal neovascular membranes (CNVM) are uncommon and may cause severe visual impairment. CNVMs may be secondary to traumatic, infectious, inflammatory, tumoral or dystrophic causes. More than $15 \%$ of CNVMs in young patients are idiopathic [2]. Etiopathogenesis of the inappropriate proliferation of the choroidal capillaries remains unclear. The focal choroiditis and choroidal ischemia may explain the capillaries neoformation but not the extensive behavior regarding the retinal and subretinal spaces [3]. The diagnosis has been made easier with the introduction of the Optical coherence tomography angiography (OCTA) that captures three-dimensional angiograms of retinal and choroidal blood vessels and allows the detection of CNVM [4]. The management of idiopathic CNVMs is still nonconsensual due to the lack of randomized pediatric trial. Recent reports showed promising stable anatomical and visual outcomes with the antivascular endothelial growth factor therapy (VEGF) $[5,6]$.

\section{Conflict of Interest: None}

\section{References}

[1] Dutta Majumder P, Ghose A, Rao C, Bhende M, Biswas J. A case of choroidal neovascular membrane in 6-year-old boy with juvenile idiopathic arthritis. J Ophthalmic Inflamm Infect. 2017; 7:18.

[2] Ong SS, Hsu ST, Grewal D, Arevalo JF, El-Dairi MA, Toth CA, Vajzovic L. Appearance of pediatric choroidal neovascular membranes on optical coherence tomography angiography. Graefes Arch Clin Exp Ophthalmol. 2020; 258:89-98.

[3] Yeo NJY, Chan EJJ, Cheung C. Choroidal Neovascularization: Mechanisms of Endothelial Dysfunction. Front Pharmacol. 2019; 10:1363.

[4] Venkat AG, Ehlers JP, Kaiser PK, Singh RP, Schachat AP, Srivastava SK, Martin DF, Rachitskaya AV. Detection of choroidal neovascular membrane beneath pigment epithelial detachment using SD-OCTA. Ophthalmic Surg Lasers Imaging Retina. 2019; 50:620-26.

[5] D'souza P, Ranjan R, Babu U, Kanakath AV, Saravanan VR. Inflammatory neovascular membrane: Long-term visua and anatomical outcomes after intravitreal anti-vascular endothelial growth factor therapy. Retina. 2018; 38:1307-15.

[6] Chrapek O, Vostrovská Z, Šínová I, Chrapková B. Treatment of idiopathic choroidal neovascular membrane with ranibizumab - our experience. Cesk Slov Oftalmol. 2019; 75:25-29. 